\title{
Sistema de Optimización en Línea y Diagnosis de Fallos para Procesos Químicos
}

Ignacio Yélamos, Sergio Ferrer, Moisès Graells y Luis Puigjaner*

Departamento de Ingeniería Química - Grupo CEPIMA, Universitat Politècnica de Catalunya, ETSEIB, Av. Diagonal 647, E-08028, Barcelona - España (e-mail: luis.puigjaner@upc.edu)

* autor a quien debe ser dirigida la correspondencia

\section{Resumen}

En este artículo, la técnica Evolución en Tiempo Real (ETR) es aplicada a la optimización en línea de una columna de destilación multicomponente. Un Sistema de Diagnóstico de Fallos (SDF) dentro de un módulo de supervisión es responsable de la gestión de cualquier incidencia (fallos o perturbaciones) que ocurra en la planta, consiguiéndose un funcionamiento en línea más robusto. La implementación de este módulo supervisado (ETRS) se ha llevado a cabo usando Matlab® y el simulador comercial Hysys ${ }^{\circledR}$, aprovechando las posibilidades de comunicación existentes entre ambos. Se tratan diferentes incidencias, involucrando diferentes fuentes y tipos de perturbaciones. Los resultados de la ETRS son comparados con los que se obtuvieron mediante la Optimización en Tiempo Real clásica.

Palabras clave: optimización, evolución en tiempo real, diagnosis de fallos, procesos químicos

\section{An On-line Optimization and Fault Diagnosis System for Chemical Processes}

\begin{abstract}
In this paper, the Real Time Evolution technique (RTE) is applied to the on-line optimization of a multicomponent distillation column. A Fault Diagnosis System (FDS) implemented within a supervisory module is responsible for handling incidences (faults or disturbances) occurring in the plant, achieving more robust on-line performance. The implementation of the supervised scheme (SRTE) has been performed using Matlab ${ }^{\circledR}$ and the commercial simulation software Hysys ${ }^{\circledR}$, taking advantage of their communication capabilities. Different possible plant incidences are considered involving different sources and types of disturbances. Results of SRTE are compared with those obtained using the standard Real Time Optimization approach.
\end{abstract}

Keywords: optimization, real time optimization, fault diagnosis, chemical processes 


\section{INTRODUCCIÓN}

La optimización en línea es un aspecto fundamental en las actuales plantas químicas que permite dar una respuesta eficaz ante perturbaciones mejorando la economía del proceso (Huang et al., 2000). La técnica de Evolución en Tiempo Real (Sequeira et al., 2002) propone un nuevo enfoque en el área de optimización en línea que ciertamente supera las limitaciones encontradas por el enfoque clásico (Marlin y Hrymak, 1997). El estado estacionario requerido en cualquier proceso dinámico necesita de un profundo filtrado y conduce generalmente a largos tiempos de espera. Además, la exigencia de modelos del proceso detallados (Yip y Marlin, 2004) y la necesidad de técnicas para el ajuste de las trayectorias de las variables, no disponibles en la mayoría de los casos, hacen de esta técnica una metodología incómoda y difícil de implementar.

Este trabajo desarrolla un sistema de gestión de perturbaciones en planta. Por tanto, la aportación se puede enmarcar en la aplicación práctica de una filosofía de integración de módulos informáticos para la mejora de la producción, que ha experimentado un gran auge en los últimos años y que ha sido destacada teóricamente en la literatura reciente (Venkatasubramanian y Yin, 2003). De esta forma, se propone un modelo de la Evolución en Tiempo Real Supervisada por un sistema de diagnóstico de fallos y aplicado a una columna de destilación multicomponente. Mediante esta combinación, el sistema opera bajo un marco mucho más robusto capaz de distinguir entre incidencias que pueden requerir un procedimiento de optimización en tiempo real (perturbaciones), e incidencias más serias (fallos) que requieran de medidas más drásticas para mantener la estabilidad y la seguridad del proceso. Esta nueva metodología decide continuamente si operar bajo la acción de la Evolución en Tiempo Real Supervisada o aplicar medidas alternativas en función de la incidencia que esté ocurriendo en cada momento.

\section{EVOLUCIÓN EN TIEMPO REAL}

La Evolución en Tiempo Real (ETR) responde rápidamente a las perturbaciones mediante un ajuste continuo de las variables de decisión en el estado estacionario. En este sentido, los parámetros cambian suave, pero continuamente, hacia los objetivos marcados sin llevar a cabo una optimización formal. De esta forma, la ETR es capaz de tratar con perturbaciones continuas debido a que no necesita alcanzar el estado estacionario para lanzar el mecanismo de mejora, consiguiendo así un beneficio claro en el funcionamiento global del proceso. La actualización del modelo sólo se realiza cuando se detecta un nuevo estado estacionario.

Esta metodología tiene cuatro pilares básicos: un algoritmo de mejora, una vecindad de búsqueda, un tiempo entre las distintas ejecuciones y un modelo de estado estacionario. El algoritmo de mejora se basa en el modo en que los nuevos puntos cercanos al punto de trabajo actual son seleccionados. La vecindad se define como el máximo cambio permitido en las variables del proceso evaluadas. Comparado con la complejidad que supone en los problemas de optimización formal el manejo de restricciones, la ETR identifica y descarta automáticamente direcciones infactibles mediante el algoritmo de mejora. Por último, para aplicar exitosamente la ETR se requerirá una selección y ajuste de estos parámetros (Sequeira et al., 2004) .

\section{MÓDULO SUPERVISOR}

Este módulo de supervisión es el núcleo fundamental del sistema de respuesta frente a desviaciones del estado normal de la planta y de su buen funcionamiento depende la fiabilidad y seguridad del proceso. El módulo supervisor organiza el funcionamiento global de la planta distinguiendo las incidencias que pueden aparecer en planta. Aquellas que afectan al beneficio económico de la planta directamente se clasifican como perturbaciones del sistema, para las que se considera acertado la activación de la Evolución en Tiempo Real Supervisada (ETRS). Por otro lado, aquellas incidencias que suponen un riesgo para la seguridad y el correcto funcionamiento del proceso son clasificadas como fallos. Este tipo de incidencias pueden requerir distintas respuestas del módulo supervisor, tales como la activación de medidas correctoras que prevengan situaciones más críticas, alarmas y gestión eventual de un paro controlado de la planta o equipo afectado. Esta clasificación entre incidencias y fallos proviene de un estudio previo del 
proceso que nos permite catalogar la gravedad de distintas perturbaciones que surgieron en el pasado (de las cuales se tienen datos) o que pudieran surgir en el futuro (para las cuales se puedan obtener datos por simulación).

La detección de las posibles incidencias se lleva a cabo en un módulo inicial de detección mediante la metodología del análisis multiescala de componentes principales (AMECP) descrita por Bakshi (1998), que se basa en el análisis de componentes principales (ACP) que es uno de los métodos más extendidos en el campo de las técnicas estadísticas multivariables para detección de fallos (Kourti y MacGregor, 1995). A través de esta estructura de detección, se combinan las capacidades del ACP para extraer las relaciones entre las distintas variables monitorizadas y la de las onditas ("wavelets" en terminología inglesa) para extraer las características esenciales de las superfluas para cualquier tipo de señal. En la práctica, la detección de una incidencia se establece cuando la suma del error cuadrático (SEC) evaluado mediante la comparación de las señales y el modelo de AMECP, excede el límite normal en tres muestreos consecutivos. Este error cuadrático se evalúa de la siguiente forma:

$$
S E C=\sum_{i=1}^{n}\left(x_{i}-x_{i}^{*}\right)^{2}
$$

donde $x_{i}$ es el valor de la variable $i$ en un instante de tiempo y $x_{i}^{*}$ es la estimación del modelo AMECP para la variable i en el mismo instante de tiempo. $\mathrm{n}$ es el número total de variables involucradas. El aislamiento y diagnosis de los fallos se realiza en un módulo de diagnóstico diferente, que consiste en una red neuronal unidireccional entrenada mediante un algoritmo de retropropagación con datos históricos de fallos y perturbaciones ocurridos en el pasado o previamente simulados. El entrenamiento y testeo de la red se desarrollaron con los correspondientes conjuntos de entrenamiento, validación y testeo para garantizar las capacidades de generalización de la red.

\section{ARQUITECTURA}

La figura 1 muestra el esquema del módulo supervisor que decide en cada momento si los datos recibidos de planta corresponden a un comportamiento normal del sistema. Cuando aparece un evento anormal, este módulo decidirá si la incidencia es crítica para el buen funcionamiento de la planta (fallo) o si por el contrario es susceptible de ser optimizada (perturbación). En este caso, la ETRS es activada automáticamente por el módulo supervisor. El tiempo para una diagnosis correcta deber ser reducido al máximo para aprovechar los beneficios de una reacción temprana del módulo de optimización.

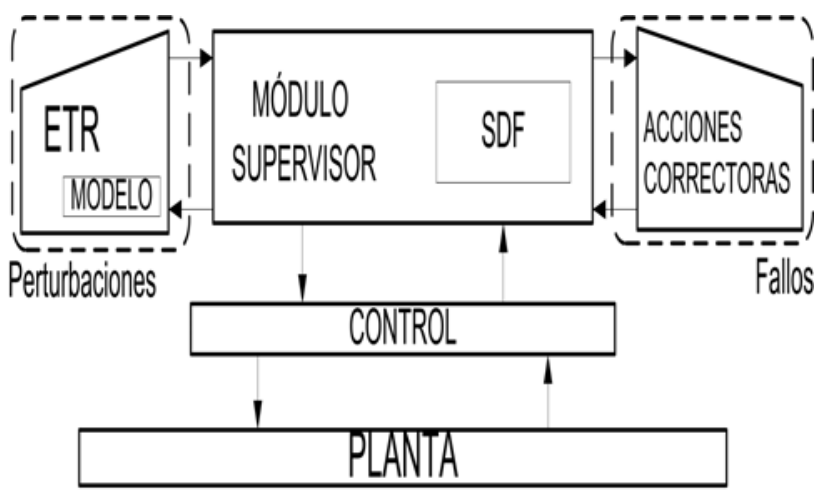

Fig. 1: Esquema del módulo supervisor

En primer lugar, las condiciones actuales de la planta son recopiladas y aplicadas al modelo de estado estacionario. Con este modelo, el algoritmo de mejora explora los alrededores de los valores actuales de las variables de decisión, evaluando la función objetivo resultante. Se elige entonces la combinación que supone una mayor mejora del valor de la función objetivo y se aplica inmediatamente en la planta a través del sistema de control. Cuando la planta alcanza sus condiciones óptimas de operación y no ocurren más perturbaciones, la ETRS se desactiva y no ejerce más acciones. Si un fallo es diagnosticado, el módulo supervisor mostrará alarmas al operador y dependiendo de la naturaleza del fallo, también podría ejecutar un protocolo previamente diseñado, consistente en una serie de acciones correctoras.

La implementación de este sistema se ha realizado mediante una función de Matlab ${ }^{\circledR}$ que funciona como un sistema coordinador estableciendo la comunicación entre la planta (simulación de Hysys ${ }^{\circledR}$ en dinámico), el modelo estacionario (Hysys ${ }^{\circledR}$ en estado estacionario), el algoritmo de optimización (en 
Matlabß) y el módulo de supervisión (también en Matlab $\left(\right.$ ). La comunicación entre Matlab ${ }^{\circledR}$ y Hysys ${ }^{\circledR}$ se realiza por medio de la tecnología COM.

\section{CASO DE ESTUDIO}

En este trabajo se ha escogido como caso de estudio la columna de destilación multicomponente que aparece en la sección de entrenamiento de la página de AspenTech ${ }^{\circledR} \quad$ (http://support.aspentech.com) para facilitar la reproducibilidad de los resultados. Esta columna es un desbutanizador de quince etapas, alimentado por dos corrientes consistentes en una mezcla de hidrocarburos ligeros. La columna ha sido simulada en Hysys ${ }^{\circledR}$ en estado estacionario (modelo para la ETRS), mientras que la simulación dinámica de esta columna, también en Hysys ${ }^{\circledR}$ (figura 2), se toma como la planta que se pretende optimizar en línea. Se ha considerado una función objetivo instantánea (IOF) que tiene en cuenta los costes de las corrientes y la energía para evaluar el funcionamiento de la planta en cada momento. La expresión de esta función objetivo instantánea en unidades monetarias por tiempo sería la siguiente:

$\operatorname{IOF}(t)=$

Flujo C4 y Ligeros · Precio Butane Product

+ Flujo C5+.Precio Liquid Product

- Flujo Feed1.Precio Feed1

- Flujo Feed2 - PrecioFeed2

- Calor Condensador · Precio Eliminar Calor

- Calor Caldera.Precio Aportar Calor

, u.m. / tiempo

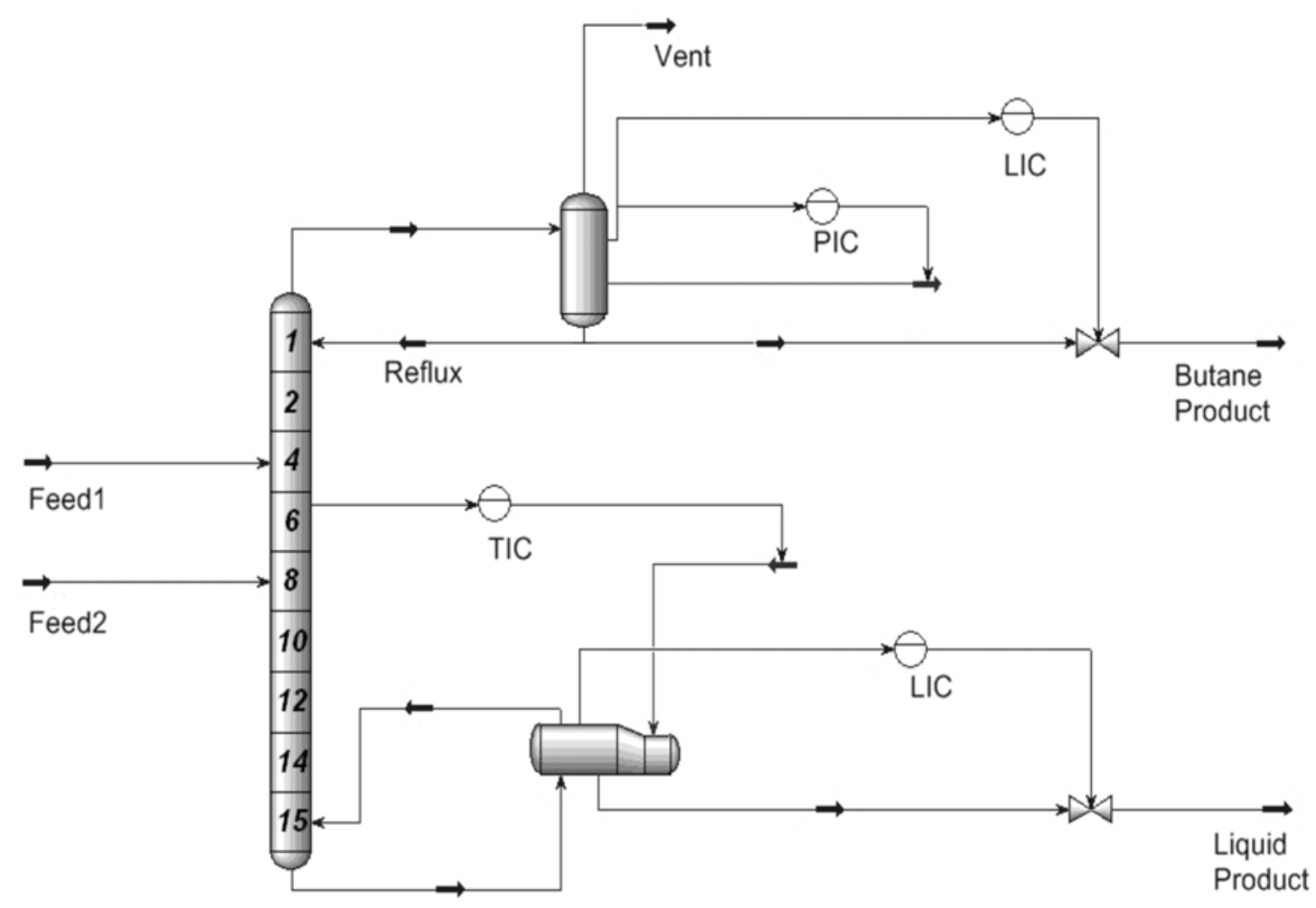

Fig. 2: Diagrama de flujo del desbutanizador

Además, se emplea una función objetivo media (MOF) que tiene en cuenta el beneficio acumulado y que se define como:

$\operatorname{MOF}(t)=\frac{\int_{t_{0}}^{t} I O F(t) d t}{t-t_{0}}$ donde $t_{0}$ y $t$ corresponden al tiempo inicial y final de evaluación, respectivamente.

\section{RESULTADOS}

En este caso de estudio, las variables de decisión son los valores de los puntos de consigna de uno de los controladores 
(temperatura del sexto plato) y la razón de reflujo de la columna. La ETR requiere una sintonización de sus parámetros en función del proceso considerado. Aquí se ha considerado un cambio máximo en las variables de decisión del $0.3 \%$ alrededor del valor actual $(2.78 \mathrm{~kg} / \mathrm{s})$ y un intervalo de 50 segundos entre las ejecuciones sucesivas, que mediante un proceso de sintonización previo acabó proporcionando resultados satisfactorios. A continuación se mostrarán dos incidentes; una perturbación y un fallo, que servirán para demostrar el funcionamiento de este módulo de optimización supervisado.

Incidente 1. Cuando se diagnostica una caída en el caudal de alimentación "Feed1" (-20\%), el módulo de supervisión lo clasifica como perturbación y automáticamente lanza la optimización mediante la ETRS. La figura 3 muestra como cuando el incidente es detectado mediante el parámetro estadístico de detección SEC y diagnosticado por el sistema de diagnosis, la ETRS es activada, consiguiéndose mejores resultados que usando la optimización en tiempo real (OTR) clásica que debe esperar a que se alcance el estado estacionario para su activación.
Cabría hacer notar que las líneas rectas punteada y sólida que aparecen en la representación del SEC con el tiempo en la figura 3 corresponden a los límites de detección, percentiles 95 y 99 del SEC respectivamente, para valores del SEC en funcionamiento normal procedentes de datos históricos de la planta. Como se puede ver en los gráficos conjuntos, tras tres muestreos consecutivos del SEC superando el límite mayor de detección (percentil 99), el incidente se detecta y se diagnostica, por lo que en ese momento se activan las medidas correctoras convenientes, en este caso, optimización mediante ETRS.

Incidente 2. En esta columna, una subida el caudal de alimentación de la corriente "Feed2" se considera un fallo que exige la acción de medidas correctoras, en este caso pasar la columna a reflujo total, hasta que el incidente sea subsanado (ver figura 4). Se comprueba como una vez corregida la fuente de error, previo diagnóstico, la función objetivo y el estadístico de detección vuelven a los niveles de condición normal de operación a un tiempo de simulación de 10000 segundos aproximadamente.

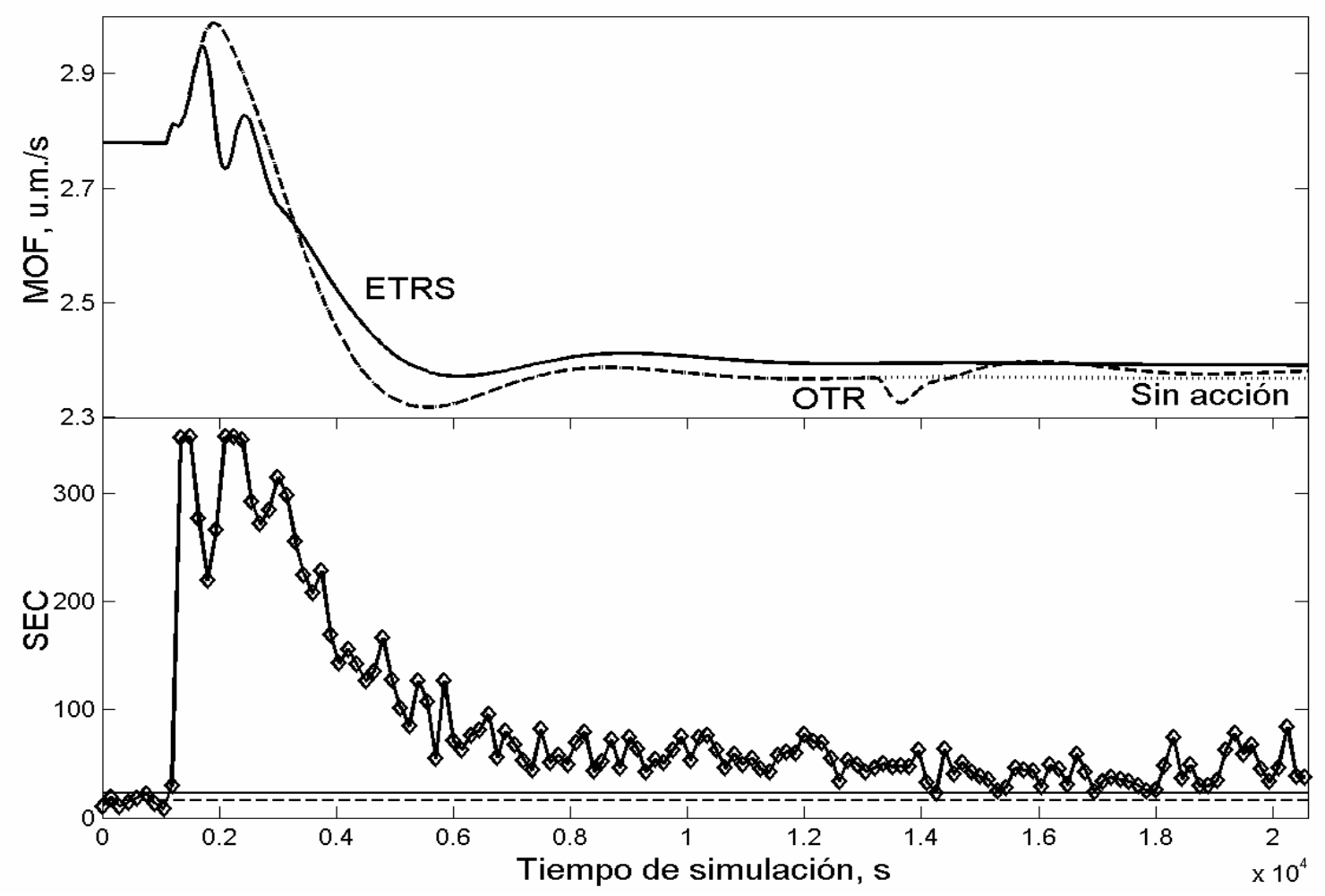

Fig. 3: Representación de la MOF y del SEC para el incidente 1. 


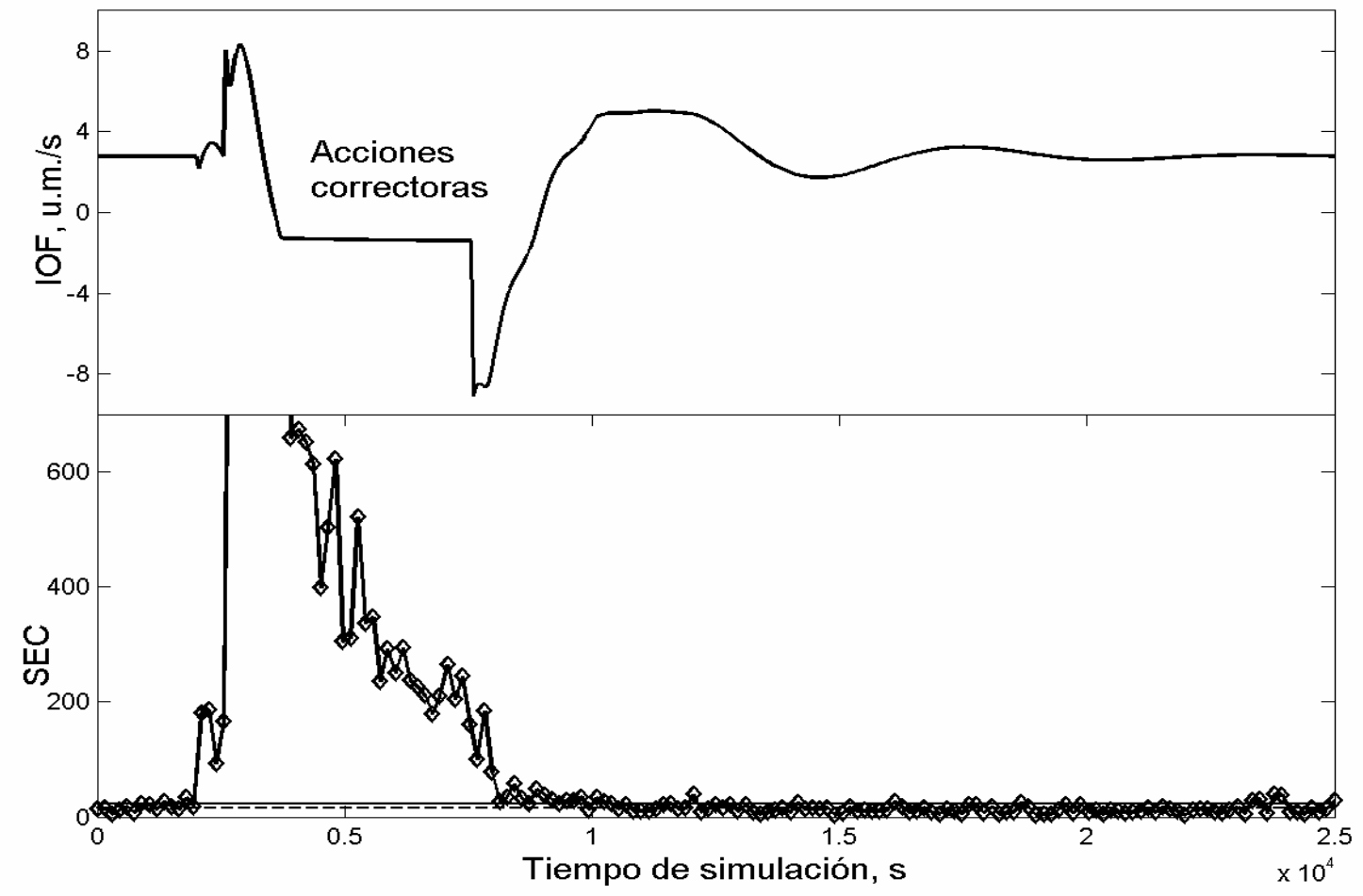

Fig. 4: Representación de la IOF y del SEC para el incidente 2.

\section{CONCLUSIONES}

En este trabajo se ha propuesto un modulo de control supervisor compuesto por un sistema de diagnóstico de fallos que está integrado con una técnica de optimización en línea. Esta optimización en línea supervisada aprovecha las características de la técnica de la Evolución en Tiempo Real consiguiendo crear un sistema mucho más robusto. Además, este módulo de supervisión proporciona más información a los operadores de planta, lo que supone una mayor base de conocimiento que será crucial en el proceso de toma de decisiones. La arquitectura propuesta ha sido aplicada a una columna de destilación multicomponente.

\section{AGRADECIMIENTOS}

A la Generalitat de Catalunya y al Ministerio de Educación y Ciencia de España por las becas FI y FPI. A la ayuda económica recibida por los proyectos: MRTN-CT-2004512233, RFC-CR-04006, INCO-CT-2005013359.

\section{REFERENCIAS}

Bakshi, B.R., Multiscale PCA with Application to Multivariate Statistical Process Monitoring, AIChE J.: 44 (7), 1596-1610 (1998)
Huang, Y., Reklaitis, G.V. y Venkatasubramanian $V$., Dynamic optimization based fault accommodation, Comput. Chem. Eng.: 24, 439444 (2000).

Kourti, T. y MacGregor, J.F., Process analysis, monitoring and diagnosis, using multivariate projection methods, Chemometrics and Intelligent Laboratory Systems: 28, 3-21 (1995)

Marlin, T.E. y Hrymak, A.N., Real-time optimization of continuous processes. AIChE Symp. Ser.: 93, 156-164 (1997)

Sequeira, S., Graells, M. y Puigjaner, L., RealTime Evolution for On-line Optimization of Continuous Processes, Ind. Eng. Chem. Res.: 41, 1815-1825 (2002)

Sequeira, S., Herrera, M., Graells, M. y Puigjaner, L., On-line process optimization: parameter tuning for the real time evolution (RTE) approach, Comput. Chem. Eng.: 28, 661672 (2004).

Venkatasubramanian, V., Rengaswamy, R., Kavury, S.N. y Yin, K., A review of process fault detection and diagnosis: Part III: Process history based methods, Comput. Chem. Eng.: $27,327-$ 346, (2003).

Yip, W.S. y Marlin, T.E., The effect of model fidelity on real-time optimization performance, Comput. Chem. Eng.: 28, 267-280 (2004).

Información Tecnológica - Vol. 18 No 2 - 2007 